\title{
Enhancements After Premium IOL Cataract Surgery: Tips, Tricks, and Outcomes
}

\author{
Mark Packer
}

Published online: 12 December 2013

(c) Springer Science + Business Media New York 2013

\begin{abstract}
Surgeons utilize enhancement procedures following premium IOL cataract surgery to reduce or eliminate residual refractive error and improve spectacle independence for their patients. The decision to perform an enhancement and the timing of enhancement surgery are often highly individualized and depend on the expectations and specific goals of the patient. Surgeons can maximize outcomes by understanding the multiple factors impacting patient satisfaction, maintaining a commitment to effective counseling, performing meticulous pre-operative diagnostic testing, adhering to the highest standards of surgical practice and honestly evaluating their surgical results.
\end{abstract}

Keywords Cataract - Intraocular lens (IOL) - Refractive error · LASIK · Piggyback IOL · Toric IOL · Presbyopiacorrecting IOL $\cdot$ Accommodating IOL $\cdot$ Refractive surgery · Enhancement procedure

\section{Introduction}

To enhance means to improve or make better. Enhancements improve the outcomes of premium IOL cataract

\footnotetext{
M. Packer

Bowie Vision Institute, 14999 Health Center Dr, Suite 108, Bowie, MD 20716, USA

M. Packer

Mark Packer MD Consulting, Inc., \% WTAS LLC, 777 S.

Flagler Drive, Suite 1700-W, West Palm Beach, FL 33401, USA

M. Packer $(\bowtie)$

Oregon Health \& Science University, Portland, OR, USA

e-mail: mark@markpackerconsulting.com
}

surgery by reducing the need for spectacle correction and thereby increasing patient satisfaction.

Premium IOL cataract surgery has two integral components. The first component consists of cataract extraction and intraocular lens implantation, a procedure that removes the cataractous lens and minimizes postoperative spherical refractive error, i.e., myopia and hyperopia, by means of proper IOL power calculation. The second component consists of further refractive correction to reduce or eliminate the need for postoperative spectacle correction and usually involves additional out-of-pocket expense, although the financial aspects of these surgical procedures vary by region and country.

\section{Premium IOL Cataract Surgery}

Premium IOL cataract surgery specifically requires the reduction or elimination of postoperative astigmatism and/or presbyopia. The reduction of postoperative higher order aberrations by means of aspheric intraocular lens implantation is not generally considered to be premium IOL cataract surgery, although some surgeons do consider the selection of aspheric IOLs based on preoperative corneal wavefront measurements to be premium IOL cataract surgery [1].

Enhancement procedures generally target suboptimal results in the refractive component of premium IOL cataract surgery, i.e., residual refractive error or presbyopia, and may sometimes also impact higher order aberrations or binocularity. Procedures undertaken to mitigate adverse events resulting from cataract surgery are not considered enhancements. For example, piggyback IOL implantation performed to decrease negative dysphotopsia [2,3] does not represent an enhancement procedure; however, piggyback IOL implantation performed to decrease hyperopia, myopia, or astigmatism does. 
The decision to proceed with enhancement is generally complex, involving the patient's current level of satisfaction with his or her degree of spectacle independence, the tolerance of blur due to refractive error (including the balance between the spherical and cylindrical components of the refraction), the acceptance of additional surgical risk, and the degree of enthusiasm for the potential benefits of enhancement surgery. The decision may also depend on the patient's financial responsibility, if any, for the additional cost of an enhancement procedure.

In addition, postoperative satisfaction usually hinges to some extent on an implicit comparison with the patient's preoperative state, including both the degree of preoperative dependence on spectacles and the expected degree of postoperative freedom from spectacles. The process by which patients develop expectations for the results of their surgery represents a major variable in their degree of satisfaction with the results and, therefore, in the potential intensity of their desire for enhancement procedures.

\section{Counseling}

While surgical and technical expertise and skill form the foundation of successful premium IOL surgery, counseling and informed consent comprise its indispensible bulwark. Counseling provides the patient with reasonable expectations for spectacle independence and incidence of adverse events. Informed consent demonstrates the patient's understanding and acceptance of the expected benefits and potential risks. These elements-surgical competence and effective communication-determine the frequency of enhancement procedures in any given surgeon's practice.

Reasonable expectations for outcomes can be gleaned from the US FDA Summary of Safety and Effectiveness Data for each particular premium IOL. Care must be taken in applying these data outside of the confines of a controlled clinical investigation, however, since a given patient considering surgery may or may not fit the inclusion and exclusion criteria employed in the trial. For example, in the Tecnis Multifocal (Abbott Medical Optics, Santa Ana, CA) clinical trial, 257 of $292(88.0 \%)$ subjects at 4-6 months postoperatively stated that they were completely spectacle independent, that they "never wore glasses." At that same time point, $255(87.3 \%)$ stated they would choose the same IOL if they had it to do over again [4]. However, potential subjects with greater than 1 diopter of preoperative keratometric astigmatism were excluded from the trial, and keratorefractive correction of astigmatism was not permitted during the trial. Therefore, one can surmise that, in order to achieve similar rates of spectacle independence and satisfaction to those demonstrated by the FDA reported data, one must either restrict patients to a maximum of 1 diopter of corneal cylinder or perform ancillary procedures in order to reduce pre-existing corneal cylinder to that level.

Reporting personal experience provides a more complete picture for patients considering surgery. In order to optimize results, surgeons should keep records of their outcomes, including enhancement rates. Patients should be informed pre-operatively of the chance they would need a second procedure in order to achieve spectacle independence.

If a patient is dissatisfied with the results of surgery, the surgeon should first ascertain the nature of the dissatisfaction. Blur due to uncorrected residual refractive error becomes the purview of enhancement procedures. If manifest refraction, with the phoropter, a pair of trial frames, or even a temporary pair of prescription spectacles, eliminates the blur and thus confirms that refractive error has caused the dissatisfaction, an enhancement procedure should be considered.

If manifest refraction does not remove the undesirable optical effect, then enhancement probably won't resolve the patient's dissatisfaction. A host of other optical problems may accompany premium IOL implantation, including the full range of multifocal dysphotopsia, negative dysphotopsia, decreased contrast sensitivity due to multifocal optics, edge glare due to decentered IOLs and chromatic complaints due to the spectral transmission of particular IOLs. None of these issues can be ameliorated by refractive enhancement.

Regardless of the nature of the dissatisfaction, the patient deserves as full an explanation as possible regarding its source. Residual spherical refractive error may simply be due to the empirical, statistical nature of IOL power calculation formulas. Measurement, calculation and implantation may have proceeded flawlessly, yet a 1 diopter error may still have occurred. On the other hand, a relatively small pupil may have resulted in a smaller than planned capsulotomy, resulting in a posterior shift in Effective Lens Position and residual hyperopic refractive error. An error in astigmatic correction may have resulted from measuring only the anterior cornea. Alternatively, a toric IOL may have rotated 10 degrees after implantation.

Limitations of IOL technology may also play a role in a patient's dissatisfaction. Relatively blurred vision when viewing a computer screen at $65 \mathrm{~cm}$ may be the expected result of diffractive multifocal IOL implantation, and relatively blurred vision at $30 \mathrm{~cm}$ may be the expected result of hinged single-optic accommodative IOL implantation. These are limitations of the optical design of these IOLs, and should have been thoroughly explained to the patient prior to surgery. They are not indications for enhancement procedures.

If post-operative refractive error is the source of dissatisfaction, the surgeon should pursue a relentless investigation to determine its source, and give a complete 
explanation to the patient. Once a patient knows that her surgeon understands the cause of her dissatisfaction, she will be greatly relieved, and will tend to join a therapeutic alliance to reach a resolution.

\section{Timing}

Usually, enhancement must wait for stability of the postoperative refraction. However, significant refractive surprise, whether involving sphere or cylinder, may represent an exception to this rule. Management of a grossly incorrect IOL power or a large degree of axis rotation (for a toric IOL) should proceed swiftly. The root cause of the error must be determined so that the appropriate corrective measures are taken.

Patients with a history of keratorefractive surgery prior to cataract surgery represent a special case. Larger postoperative refractive errors may be expected in this group, and patients with a history of incisional surgery (such as radial keratotomy) must exercise patience until reasonable stability of their refraction is achieved.

\section{Choice of Procedure}

Enhancement procedures may take many forms. Clinical experience and creativity help to determine the optimal approach for each patient. Personalizing the procedure will likely optimize the outcome.

Laser keratorefractive surgery, i.e., LASIK or PRK, often comes to mind first in the treatment of residual refractive error after premium IOL cataract surgery. The relatively small amounts of correction needed, compared to those for which these procedures are used in virgin eyes, suggest there is a high probability of success in achieving the targeted refractions. Incisional procedures such as Limbal Relaxing Incisions can also serve for small amounts of cylindrical correction. However, the greater prevalence of ocular surface disease among patients with cataracts suggests caution in employing the keratorefractive approach. Dry eye can pose significant risk for decreased best-corrected visual acuity following these procedures. Treatment of pre-existing external disease, including blepharitis and tear film insufficiency, represents good practice not only prior to keratorefractive procedures but also prior to premium IOL surgery itself.

Piggyback IOLs offer another pathway to refractive correction and have the advantage of not compromising the ocular surface to the same degree as keratorefractive procedures. However, the choice of IOL is limited in the US to spherical correction (outside the US, toric piggyback IOLs are available). In addition, placing a piggyback IOL represents a second intraocular procedure and carries a significantly different risk profile from LASIK. Nevertheless, a piggyback IOL may serve as the best choice in a patient with dry eye, odd corneal topography or a purely spherical refractive error.

A variety of intraocular manipulations of the capsule and iris can serve to increase the effectiveness of particular premium IOLs. For example, a YAG posterior capsulotomy or selective posterior capsulotomy can affect the axial position of a single-optic, hinged accommodative, or toric accommodative IOL. Photomydriasis has shown positive results in improving the near vision with zonal refractive multifocal IOLs, and has been suggested for improving the alignment of the pupil with the ring structure of diffractive multifocal IOLs. In addition, pharmacologic constriction of the pupil may be useful for reducing higher order aberrations and increasing depth of focus in selected patients.

\section{Astigmatism Correction}

The effectiveness of toric IOLs depends on accurate measurement of corneal astigmatism, proper power selection for both sphere and cylinder, and precise alignment. Recently, the role of posterior corneal curvature has emerged as an explanation for the higher incidence of under-correction in against-the-rule astigmatism [5••]. Innovative modalities for imaging and registration have become available to replace the standard ink markings used to align toric IOLs. In addition, intraoperative aberrometry now allows measurement during surgery to increase accuracy by taking into account not only the posterior corneal contribution but also the individual effect of surgically induced astigmatism in each particular eye [6].

Crossed cylinder calculation demonstrates that a rotation of 33 degrees negates the effective correction of a toric IOL. FDA reported data and product labeling report the rotational stability of toric IOLs. For example, in the Trulign toric IOL clinical trial (Bausch \& Lomb, Aliso Viejo, CA, USA), $96.1 \%$ of eyes exhibited rotation of less than or equal to 5 degrees between the day of surgery and 4-6 months postoperatively [7]. For low toric powers, even a somewhat greater misalignment than 5 degrees may not warrant an enhancement procedure, depending on the level of patient satisfaction. Given the high degree of rotational stability, most misalignments will likely be due to errors in placement at the time of surgery rather than post-operative rotation.

In general, residual cylinder following toric IOL implantation may be due to errors in measurement, calculation or alignment. Neglect of the posterior cornea may be the most important measurement error. The relatively large standard deviations typical of Surgically Induced Astigmatism also rank high as a source of error. Calculation errors most likely result from inaccurate input; however, most formulas and calculators also contain inherent errors such as 
neglect of particular variables (e.g., the impact of the Effective Lens Position on the toric correction).

Gross errors in surgical alignment will usually be relatively easy to identify and are best corrected by IOL repositioning. Occasionally the toric markings on the IOL remain hidden behind a small pupil. In these cases the use of a gonioscopy lens can help identify the problem.

Repositioning of toric IOLs remains possible even years following implantation, as long as the capsule remains intact. Once a YAG laser has opened the posterior capsule, the risks of vitreous prolapse and retinal tear or detachment increase. Re-opening an intact fibrotic capsular bag requires careful viscodissection, particularly along the path of the haptics, using a dispersive Ophthalmic Viscosurgical Device. Certain haptic designs, especially the polyimide loops of hinged single-optic toric IOLs, resist liberation and may require amputation and IOL exchange rather than repositioning.

Early in the post-operative course, prior to capsule shrinkage at 3-6 weeks, repositioning is straightforward. The capsule is filled with cohesive OVD and the IOL is gently rotated using a hook at the haptic-optic junction. The haptics of hinged single-optic toric IOLs may be pulled centrally to insure they are free from the capsule equator prior to rotation.

While the repositioning is easily accomplished during this early period, avoiding recurrence of whatever caused the initial rotation may not be as easy. A simple surgical marking error or suboptimal alignment due to a small pupil, for example, may be easily overcome. Post-operative IOL rotation due to a relative large capsular bag, however, has the same risk the second time around. Similarly, a capsular bag with localized fibrosis around the IOL haptics may tend to defeat attempted repositioning. These cases occasionally require IOL exchange and a second enhancement procedure, which is usually keratorefractive.

Small errors in astigmatic correction may initially be addressed with a keratorefractive procedure rather than repositioning. The presence of a toric IOL is essentially ignored in determining the appropriate excimer laser treatment or Limbal Relaxing Incisions, which are based solely on the refraction. The usual cautions about ectasia and ocular surface disease must be observed. LRIs can be performed at the slit lamp in cooperative patients; a minor OR with a basic operating microscope also works well.

\section{Piggyback IOLs}

Piggyback placement of an intraocular lens can represent a successful strategy for correction of residual refractive error [8].

In the US, options for appropriate piggyback IOLs remain limited to the correction of residual spherical equivalent refractive error; outside the US, available piggyback designs include aspheric, toric and multifocal lenses. Appropriate power calculation for piggyback IOLs is based on residual refractive error, and can achieve highly accurate outcomes. Complications of piggybacking including interlenticular opacification, pigment dispersion, iridocyclitis, glaucoma and hyphema can be avoided with proper preoperative planning and IOL selection.

Successful refractive enhancement with piggyback IOLs has recently been reported in a series of 18 eyes (out of a total of 74 eyes of 41 patients) following premium IOL surgery with hinged single-optic accommodative IOL implantation (eyes with a history of previous keratorefractive surgery were excluded). Eight eyes had planned piggybacks for expected residual hyperopia and 10 eyes had unplanned piggybacks for refractive surprise [9*0].

Among the planned piggybacks the mean axial length was $20.43 \pm 0.97 \mathrm{~mm}$ (range, 18.47-21.42); the total calculated IOL power was $33.12 \pm 3.87 \mathrm{D}(29.0-41.5)$. These piggyback IOLs were implanted within three weeks of the primary surgery, and occasionally contemporaneously with implantation of the primary IOL. The eyes with unplanned piggybacks had a mean axial length of $21.46 \pm 0.57 \mathrm{~mm}$ (20.33-22.27). These piggybacks were implanted from 6 to 14 weeks after the initial surgery, once the postoperative refraction had stabilized and the need for enhancement was confirmed. In this series, axial length was the best predictor of whether or not a patient would likely need a piggyback IOL $(p<0.001)$.

IOL power calculations were performed with the HolladayR formula (Holladay IOL Consultant, Bellaire, TX, USA). This formula takes into account the A constant of the piggyback IOL and the residual refractive error (manifest refractive spherical equivalent). In contradistinction to primary IOL implantation, keratometry, axial length, lens thickness, and corneal white-to-white values are irrelevant when calculating the power of the piggyback IOL. Only the intended correction and the lens constant (representing the Effective Lens Position) are taken into account (this approach is quite familiar to surgeons who implant phakic refractive lenses, or, for that matter, to doctors who fit contact lenses). In the US, the most popular piggyback IOL is the AQ5010 (STAAR Surgical, Monrovia, CA, USA) because of its round edge, $6.3 \mathrm{~mm}$ diameter optic and $13.5 \mathrm{~mm}$ overall diameter. Outside the US, purposedesigned additive IOLs designed specifically for implantation in the ciliary sulcus are available.

In this retrospective study of piggyback IOL enhancement there was an excellent correlation between the targeted and achieved spherical equivalent for the unplanned piggyback IOLs (Pearson Correlation Coefficient $=0.87$ ). The mean uncorrected distance visual acuity improved from about $20 / 60$ to $20 / 20$ post-operatively, and the uncorrected 
Fig. 1 A patient with a history of Hexagonal Keratotomy for the correction of hyperopia developed cataracts and underwent phacoemulsification and IOL implantation elsewhere. Because the surgeon failed to take into account the inaccuracy of keratometry after keratorefractive surgery, the cataract surgery resulted in a spherical equivalent refractive surprise of $-6.5 \mathrm{D}$ with $3.0 \mathrm{D}$ of refractive astigmatism. A negatively powered piggyback IOL (CLRFLXB, -8.0 D [Abbott Medical Optics, Inc., Santa Ana, CA]) was used to correct the spherical equivalent (subsequent PRK with adjunctive mitomycin $\mathrm{C}$ corrected the residual astigmatism). a demonstrates construction of a $2.5 \mathrm{~mm}$ temporal clear corneal incision. The anterior chamber and ciliary sulcus were then filled with a cohesive OVD. b shows insertion of the piggyback IOL, with the leading haptic placed in the distal ciliary sulcus. The trailing haptic remained external to the incision $\mathbf{c}$ and was then dialed into position $\mathbf{d}$. Following aspiration of OVD the incision was sealed with stromal hydration
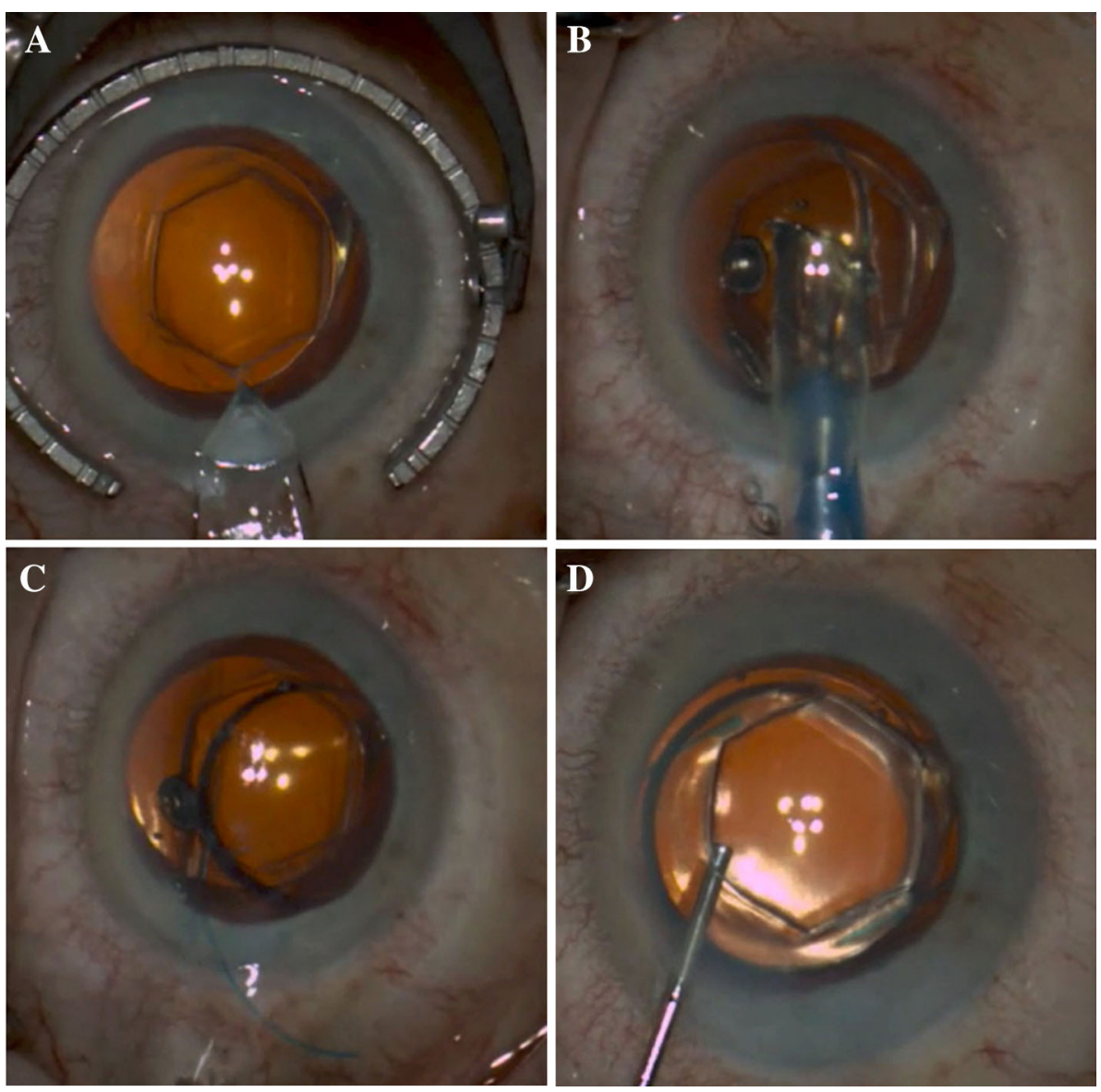

near acuity improved from about J10 to J3. This series clearly supported the use of piggybacking to improve uncorrected vision at both distance and near for patients implanted with a single optic, hinged accommodative IOL.

The advantages of using piggyback IOLs for enhancement in these cases included rapid rehabilitation, excellent predictability, and no need for an excimer laser. However, intraocular surgery carries with it greater cost than corneal refractive surgery; the correction is limited by available powers of IOLs, and, at least in the US, no IOLs suitable for piggybacking permit toric correction.

Outside the US, one example of an available additive IOL specifically designed for implantation in the ciliary sulcus of the pseudophakic eye is the Sulcoflex IOL (Rayner Intraocular Lenses, Ltd., Hove, England). It is a single-piece hydrophilic acrylic IOL that can be inserted through a $3.0 \mathrm{~mm}$ incision. The $6.5 \mathrm{~mm}$ optic and haptic edges are round. The haptic is angulated and has an undulating design to preclude rotation. A spherical monofocal version of the Sulcoflex has been implanted in the ciliary sulcus of pseudophakic eyes to correct residual ametropia [10]. Toric, multifocal and aspheric versions of the IOL are also available to correct residual astigmatism, permit presbyopia correction and reduce higher order aberrations in pseudophakic eyes [11].
Multiple peer-reviewed publications have supported the effectiveness of both primary (at the same time as the initial surgery) and secondary (delayed) placement of piggyback IOLs. Akaishi et al. [12, 13] have reported placing a silicone piggyback IOL in the sulcus to enhance correction with both the Tecnis and the ReSTOR (Alcon Surgical, Ft. Worth, TX, USA) multifocal IOLs. Recently Jin et al. [14] reported effective correction of residual astigmatism with placement of a toric IOL in the sulcus using an obliquely crossed cylinder technique. Alfonso et al. [15] have described placing diffractive multifocal IOLs in the sulcus to provide pseudoaccommodation, and Boisvert et al. [16] have developed a pediatric piggyback IOL calculator to facilitate the strategy of temporary polypseudophakia in young children by removing the anterior IOL when the eye becomes sufficiently myopic due to the expected physiologic shift in refraction.

Despite enthusiasm for piggyback IOLs, surgeons should remain aware of potential complications. For example, interlenticular opacification (ILO) has been reported with the implantation of two acrylic IOLs in the capsular bag. In general, the use of a silicone piggyback IOL placed in the sulcus is recommended to prevent the development of ILO [17]. Pigment dispersion and 
pigmentary glaucoma have been reported with placement of IOLs with sharp anterior optic edges in the ciliary sulcus $[18,19]$; hence, the requirement for rounded anterior optic edges on piggyback IOLs [20••].

An unusual complication of piggyback IOL insertion is posterior capsule rupture [21•]. Decreased elasticity of the posterior capsule may make it less resilient during intraocular irrigation for removal of OVD.

Piggyback IOLs can achieve excellent results and likely represent the best choice for correction of residual spherical ametropia in eyes with a history of prior radial keratotomy and eyes with ocular surface disease or suspicious corneal topography which are not good candidates for LASIK or PRK. When an astigmatic component is present and the eye is otherwise healthy, however, corneal refractive procedures offer an unexcelled degree of accuracy and precision in a cost-effective manner (Fig. 1).

\section{Limits of Accuracy}

Reasonable expectations set the stage for success in premium IOL surgery. Multifocal IOLs permit excellent acuity at distance and near, and spectacle independence may be achieved in up to $88 \%$ of patients following primary bilateral implantation [4]. Given this rate of success, one in ten patients may find they still need glasses for some tasks. Significant residual refractive error in this group may be reduced with enhancement procedures, thus increasing the success rate of the procedure. However, smaller amounts of refractive error may prove refractory to correction, and futile attempts to eliminate them may engender frustration and magnify dissatisfaction.

In general, \pm 0.25 diopter spherical equivalent manifest refraction and 0.50 diopter or less manifest cylinder represent a successful outcome and should not be enhanced further. These magnitudes are within the limits of precision for both keratorefractive and IOL surgery. Patients who remain dissatisfied despite multiple refractions within these limits may be experiencing surgical side effects that are not amenable to enhancement procedures. A careful examination augmented by appropriate diagnostic technology may reveal occult causative factors such as ocular motility disorders, ocular surface disease, intraocular inflammation, IOL subluxation, capsular opacification, vitreous syneresis, and epiretinal membranes or cystoid macular edema. Appropriate treatment may facilitate an improvement in symptomatology.

\section{Conclusion}

Enhancement procedures can save the day when refractive surprise threatens to mar the outcome of premium IOL surgery. However, appropriate counseling and accurate diagnosis remain critical to success. Patience often becomes the most important virtue, because victory may be achieved while simply waiting for stability.

Disclosure Dr. Packer is a Consultant for Abbott Medical Optics, Inc., Bausch \& Lomb Surgical, Inc., Lensar, Inc. Rayner Intraocular Lenses, Ltd., TrueVision Systems, Inc. and WaveTec Vision Systems, Inc.

Human and Animal Rights and Informed Consent This article does not contain any studies with human or animal subjects performed by any of the authors.

\section{References}

Papers of particular interest, published recently, have been highlighted as:

- Of importance

•• Of major importance

1. Packer M, Fine IH, Hoffman RS. Aspheric intraocular lens selection based on corneal wavefront. J Refract Surg. 2009;25: 12-20.

2. Ernest PH. Severe photic phenomenon. J Cataract Refract Surg. 2006;32(4):685-6.

3. Masket S, Fram NR. Pseudophakic negative dysphotopsia: Surgical management and new theory of etiology. J Cataract Refract Surg. 2011;37:1199-207.

4. Summary of Safety and Effectiveness Data. http://www. accessdata.fda.gov/cdrh_docs/pdf/P980040S039b.pdf. Accessed 13 Oct 2013.

5. • Koch DD, Ali SF, Weikert MP, Shirayama M, Jenkins R, Wang L. Contribution of posterior corneal astigmatism to total corneal astigmatism. J Cataract Refract Surg. 2012;38(12): 2080-7. The authors demonstrate the significance of posterior corneal astigmatism. While application of population mean values of posterior corneal astigmatism to toric IOL surgery will likely improve outcomes, further improvement depends on individual measurement.

6. Packer M. Effect of intraoperative aberrometry on the rate of postoperative enhancement: retrospective study. J Cataract Refract Surg. 2010;36(5):747-55.

7. AcrySof ${ }^{\circledR}$ IQ ReSTOR ${ }^{\circledR}$ IOL (Model SN6AD1). Directions for Use.

8. Packer M, Hoffman RS. Piggyback IOLs: strategies and considerations. Cataract Refract Surg Today Eur. 2010;5(6):18-21.

9. •- Packer M. Frequency and Risk Factor Analysis of Piggyback IOL Enhancement Following Refractive Lens Exchange With an Accommodative IOL. Refractive Surgery 2006: The Times They Are A-Changin' Sponsored by the International Society of Refractive Surgery of the American Academy of Ophthalmology (ISRS/AAO). Las Vegas, NV, 10-11 November 2006. Johnstown, PA: Conference Archives, Inc. Multimedia DVD-ROM Archive (Flash). The results of this retrospective study are summarized in the current article. Piggyback enhancement represented an effective strategy for the correction of residual spherical refractive error in this group of patients implanted with a single optic, hinged accommodative IOL.

10. Amon M, Kahraman G, Schauersberger J. "Sulcoflex (Rayner 653L), a New IOL for Implantation in the Pseudophakic Eye: 
Indications and First Results," presented at the XXIV Congress of the European Society of Cataract and Refractive Surgeons, Stockholm, September 2007. Abstract available at http://www. rayner.com/pdfs/Reference109.pdf. Accessed 27 Apr 2010.

11. Falzon K, Stewart OG. Correction of undesirable pseudophakic refractive error with the Sulcoflex intraocular lens. J Refract Surg. 2012;28(9):614-9.

12. Akaishi L, Tzelikis PF, Gondim J, Vaz R. Primary piggyback implantation using the Tecnis ZM900 multifocal intraocular lens: case series. J Cataract Refract Surg. 2007;33(12):2067-71.

13. Akaishi L, Tzelikis PF. Primary piggyback implantation using the ReSTOR intraocular lens: case series. J Cataract Refract Surg. 2007;33(5):791-5.

14. Jin H, Limberger IJ, Borkenstein AF, Ehmer A, Guo H, Auffarth GU. Pseudophakic eye with obliquely crossed piggyback toric intraocular lenses. J Cataract Refract Surg. 2010;36(3):497-502.

15. Alfonso JF, Fernández-Vega L, Baamonde MB. Secondary diffractive bifocal piggyback intraocular lens implantation. J Cataract Refract Surg. 2006;32(11):1938-43.

16. Boisvert C, Beverly DT, McClatchey SK. Theoretical strategy for choosing piggyback intraocular lens powers in young children. J AAPOS. 2009;13(6):555-7.

17. Werner L, Mamalis N, Stevens S, Hunter B, Chew JJ, Vargas LG. Interlenticular opacification: dual-optic versus piggyback intraocular lenses. J Cataract Refract Surg. 2006;32(4):655-61.
18. Chang WH, Werner L, Fry LL, Johnson JT, Kamae K, Mamalis N. Pigmentary dispersion syndrome with a secondary piggyback 3 -piece hydrophobic acrylic lens. Case report with clinicopathological correlation. J Cataract Refract Surg. 2007;33(6):1106-9.

19. Iwase T, Tanaka N. Elevated intraocular pressure in secondary piggyback intraocular lens implantation. J Cataract Refract Surg. 2005;31(9):1821-3.

20. • Chang DF, Masket S, Miller KM, Braga-Mele R, Little BC, Mamalis N, Oetting TA, Packer M. ASCRS Cataract Clinical Committee. Complications of sulcus placement of single-piece acrylic intraocular lenses: recommendations for backup IOL implantation following posterior capsule rupture. J Cataract Refract Surg. 2009;35(8):1445-58. These authors present a thorough review of the pathogenesis of pigment dispersion and other complications associated with the placement of single piece acrylic IOLs in the ciliary sulcus.

21. - Packer M. The Perils of Piggybacking. Chang DF (ed), Cataract Surgery: My Most Difficult Case. Cataract \& Refractive Surgery Today. 2009;9(7): 29-33, available at http://bmctoday.net/ crstoday/pdfs/CRST0709_05.pdf. Accessed 28 Apr 2010. This case study involving multiple surgical interventions for the attempted correction of dysphotopsia represents not only a review of potential complications but also a cautionary tale. 